KARAKAŞ A.

\title{
A NEW FACTOR THEOREM FOR GENERALIZED ABSOLUTE RIESZ SUMMABILITY
}

The aim of this paper is to consider an absolute summability method and generalize a theorem concerning $\left|\bar{N}, p_{n}\right|_{k}$ summability of infinite series to $\varphi-\left|\bar{N}, p_{n} ; \delta\right|_{k}$ summability of infinite series by using almost increasing sequence. Furthermore, it is explained that a well known result dealing with $\left|\bar{N}, p_{n}\right|_{k}$ summability is obtained when this generalization is restricted under special conditions.

Key words and phrases: summability factors, almost increasing sequence, infinite series, Hölder inequality, Minkowski inequality.

Department of Mathematics, Erciyes University, 38039, Kayseri, Turkey

E-mail: ahmetkarakas1985ehotmail.com

\section{INTRODUCTION}

A positive sequence $\left(z_{n}\right)$ is said to be almost increasing if there exists a positive increasing sequence $\left(d_{n}\right)$ and two positive constants $L$ and $M$ such that $L d_{n} \leq z_{n} \leq M d_{n}$ (see [1]).

Let $\sum a_{n}$ be a given infinite series with partial sums $\left(s_{n}\right)$. Let $\left(p_{n}\right)$ be a sequence of positive numbers such that

$$
P_{n}=\sum_{v=0}^{n} p_{v} \rightarrow \infty \quad \text { as } \quad n \rightarrow \infty, \quad\left(P_{-i}=p_{-i}=0, i \geq 1\right)
$$

The sequence-to-sequence transformation

$$
w_{n}=\frac{1}{P_{n}} \sum_{v=0}^{n} p_{v} s_{v}
$$

defines the sequence $\left(w_{n}\right)$ of the $\left(\bar{N}, p_{n}\right)$ means of the sequence $\left(s_{n}\right)$, generated by the sequence of coefficients $\left(p_{n}\right)$ (see [8]). The series $\sum a_{n}$ is said to be summable $\left|\bar{N}, p_{n}\right|_{k}, k \geq 1$, if (see [2])

$$
\sum_{n=1}^{\infty}\left(\frac{P_{n}}{p_{n}}\right)^{k-1}\left|w_{n}-w_{n-1}\right|^{k}<\infty
$$

Let $\left(\varphi_{n}\right)$ be any sequence of positive real numbers. The series $\sum a_{n}$ is said to be summable $\varphi-\left|\bar{N}, p_{n} ; \delta\right|_{k}, k \geq 1$ and $\delta \geq 0$, if (see [16])

$$
\sum_{n=1}^{\infty} \varphi_{n}^{\delta k+k-1}\left|w_{n}-w_{n-1}\right|^{k}<\infty
$$

If we take $\varphi_{n}=\frac{P_{n}}{p_{n}}$, then $\varphi-\left|\bar{N}, p_{n} ; \delta\right|_{k}$ summability is the same as $\left|\bar{N}, p_{n} ; \delta\right|_{k}$ summability (see [4]). Also, if we take $\varphi_{n}=\frac{P_{n}}{p_{n}}$ and $\delta=0$, then we get $\left|\bar{N}, p_{n}\right|_{k}$ summability. 


\section{THE KNOWN RESULT}

A well known theorem dealing with $\left|\bar{N}, p_{n}\right|_{k}$ summability factors of infinite series is given below.

Theorem 1 ([3]). Let $\left(X_{n}\right)$ be a positive non-decreasing sequence and suppose that there exists sequences $\left(\lambda_{n}\right)$ and $\left(\beta_{n}\right)$ such that

$$
\begin{gathered}
\left|\Delta \lambda_{n}\right| \leq \beta_{n}, \\
\beta_{n} \rightarrow 0 \quad \text { as } n \rightarrow \infty, \\
\sum_{n=1}^{\infty} n\left|\Delta \beta_{n}\right| X_{n}<\infty, \\
\left|\lambda_{n}\right| X_{n}=O(1) \text { as } n \rightarrow \infty .
\end{gathered}
$$

If

$$
\sum_{n=1}^{m} \frac{1}{n}\left|s_{n}\right|^{k}=O\left(X_{m}\right) \quad \text { as } \quad m \rightarrow \infty
$$

and $\left(p_{n}\right)$ is a sequence such that

$$
\begin{aligned}
P_{n} & =O\left(n p_{n}\right), \\
P_{n} \Delta p_{n} & =O\left(p_{n} p_{n+1}\right),
\end{aligned}
$$

then the series $\sum_{n=1}^{\infty} a_{n} \frac{P_{n} \lambda_{n}}{n p_{n}}$ is summable $\left|\bar{N}, p_{n}\right|_{k}, k \geq 1$.

\section{The MAIN RESUlT}

Some works dealing with generalized absolute summability methods have been done (see $[5-7,9,10,13-19])$. The aim of this paper is to generalize Theorem 1 to $\varphi-\left|\bar{N}, p_{n} ; \delta\right|_{k}$ summability using almost increasing sequence in place of positive non-decreasing sequence.

Theorem 2. Let $\left(\varphi_{n}\right)$ be a sequence of positive real numbers such that

$$
\begin{gathered}
\varphi_{n} p_{n}=O\left(P_{n}\right), \\
\sum_{n=v+1}^{m+1} \varphi_{n}^{\delta k-1} \frac{1}{P_{n-1}}=O\left(\varphi_{v}^{\delta k} \frac{1}{P_{v}}\right) \text { as } m \rightarrow \infty .
\end{gathered}
$$

Let $\left(X_{n}\right)$ be an almost increasing sequence. If conditions (1)-(4), (6)-(7) of the Theorem 1 and

$$
\sum_{n=1}^{m} \varphi_{n}^{\delta k} \frac{\left|s_{n}\right|^{k}}{n}=O\left(X_{m}\right) \text { as } m \rightarrow \infty
$$

are satisfied, then the series $\sum_{n=1}^{\infty} a_{n} \frac{p_{n} \lambda_{n}}{n p_{n}}$ is summable $\varphi-\left|\bar{N}, p_{n} ; \delta\right|_{k}, k \geq 1$ and $0 \leq \delta k<1$. 
We need the following lemmas for the proof of Theorem 2.

Lemma 1 ([11]). Under the conditions on $\left(X_{n}\right),\left(\beta_{n}\right)$ and $\left(\lambda_{n}\right)$ as taken in the statement of the theorem, we have that

$$
\begin{gathered}
n X_{n} \beta_{n}=O(1) \text { as } n \rightarrow \infty, \\
\sum_{n=1}^{\infty} \beta_{n} X_{n}<\infty .
\end{gathered}
$$

Lemma 2 ([12]). If the conditions (6) and (7) of Theorem 1 are satisfied, then $\Delta\left(\frac{P_{n}}{n p_{n}}\right)=O\left(\frac{1}{n}\right)$. Remark 1 ([3]). It should be noted that, from the hypotheses of Theorem 1, $\left(\lambda_{n}\right)$ is bounded and $\Delta \lambda_{n}=O(1 / n)$.

\section{PROOF OF THEOREM 2}

Proof. Let $\left(J_{n}\right)$ indicate $\left(\bar{N}, p_{n}\right)$ means of the series $\sum_{n=1}^{\infty} a_{n} \frac{P_{n} \lambda_{n}}{n p_{n}}$. Then, for $n \geq 1$, we obtain

$$
\bar{\Delta} J_{n}=\frac{p_{n}}{P_{n} P_{n-1}} \sum_{v=1}^{n} P_{v-1} \frac{a_{v} P_{v} \lambda_{v}}{v p_{v}} .
$$

Applying Abel's formula, we get

$$
\begin{aligned}
\bar{\Delta} J_{n} & =\frac{s_{n} \lambda_{n}}{n}+\frac{p_{n}}{P_{n} P_{n-1}} \sum_{v=1}^{n-1} \frac{P_{v+1} P_{v} \Delta \lambda_{v}}{(v+1) p_{v+1}} s_{v}+\frac{p_{n}}{P_{n} P_{n-1}} \sum_{v=1}^{n-1} P_{v} \lambda_{v} s_{v} \Delta\left(\frac{P_{v}}{v p_{v}}\right)-\frac{p_{n}}{P_{n} P_{n-1}} \sum_{v=1}^{n-1} P_{v} \lambda_{v} s_{v} \frac{1}{v} \\
& =J_{n, 1}+J_{n, 2}+J_{n, 3}+J_{n, 4} .
\end{aligned}
$$

For the proof of Theorem 2, it is sufficient to show that

$$
\sum_{n=1}^{\infty} \varphi_{n}^{\delta k+k-1}\left|J_{n, r}\right|^{k}<\infty, \quad \text { for } \quad r=1,2,3,4
$$

By using Abel's formula, we have

$$
\begin{aligned}
\sum_{n=1}^{m} \varphi_{n}^{\delta k+k-1}\left|J_{n, 1}\right|^{k} & =O(1) \sum_{n=1}^{m} \varphi_{n}^{\delta k+k-1} \frac{1}{n^{k}}\left|\lambda_{n}\right|^{k-1}\left|\lambda_{n}\right|\left|s_{n}\right|^{k}=O(1) \sum_{n=1}^{m} \varphi_{n}^{\delta k}\left|\lambda_{n}\right| \frac{\left|s_{n}\right|^{k}}{n} \\
& =O(1) \sum_{n=1}^{m-1} \Delta\left|\lambda_{n}\right| \sum_{v=1}^{n} \varphi_{v}^{\delta k} \frac{\left|s_{v}\right|^{k}}{v}+O(1)\left|\lambda_{m}\right| \sum_{n=1}^{m} \varphi_{n}^{\delta k} \frac{\left|s_{n}\right|^{k}}{n} \\
& =O(1) \sum_{n=1}^{m-1} \beta_{n} X_{n}+O(1)\left|\lambda_{m}\right| X_{m}=O(1) \quad \text { as } \quad m \rightarrow \infty,
\end{aligned}
$$

by virtue of (1), (4), (6), (8), (10) and (12).

Now, using Hölder's inequality and (1), (6), (8), we obtain

$$
\begin{aligned}
\sum_{n=2}^{m+1} \varphi_{n}^{\delta k+k-1}\left|J_{n, 2}\right|^{k} & =O(1) \sum_{n=2}^{m+1} \varphi_{n}^{\delta k+k-1}\left(\frac{P_{n}}{P_{n} P_{n-1}}\right)^{k}\left(\sum_{v=1}^{n-1} P_{v}\left|\Delta \lambda_{v}\right|\left|s_{v}\right|\right)^{k} \\
& =O(1) \sum_{n=2}^{m+1} \varphi_{n}^{\delta k-1} \frac{1}{P_{n-1}^{k}}\left(\sum_{v=1}^{n-1} P_{v}\left|\Delta \lambda_{v}\right|\left|s_{v}\right|\right)^{k} \\
& =O(1) \sum_{n=2}^{m+1} \varphi_{n}^{\delta k-1} \frac{1}{P_{n-1}}\left(\sum_{v=1}^{n-1} P_{v} \beta_{v}\left|s_{v}\right|^{k}\right) \times\left(\frac{1}{P_{n-1}} \sum_{v=1}^{n-1} P_{v} \beta_{v}\right)^{k-1} .
\end{aligned}
$$


Again, using Abel's formula and (3), (9)-(12), we have

$$
\begin{aligned}
\sum_{n=2}^{m+1} \varphi_{n}^{\delta k+k-1}\left|J_{n, 2}\right|^{k}= & O(1) \sum_{n=2}^{m+1} \varphi_{n}^{\delta k-1} \frac{1}{P_{n-1}} \sum_{v=1}^{n-1} P_{v} \beta_{v}\left|s_{v}\right|^{k}=O(1) \sum_{v=1}^{m} P_{v} \beta_{v}\left|s_{v}\right|^{k} \sum_{n=v+1}^{m+1} \varphi_{n}^{\delta k-1} \frac{1}{P_{n-1}} \\
= & O(1) \sum_{v=1}^{m} \varphi_{v}^{\delta k} \frac{\left|s_{v}\right|^{k}}{v} v \beta v=O(1) \sum_{v=1}^{m-1} \Delta\left(v \beta_{v}\right) \sum_{r=1}^{v} \varphi_{r}^{\delta k} \frac{\left|s_{r}\right|^{k}}{r} \\
& +O(1) m \beta_{m} \sum_{v=1}^{m} \varphi_{v}^{\delta k} \frac{\left|s_{v}\right|^{k}}{v}=O(1) \sum_{v=1}^{m-1} \Delta\left(v \beta_{v}\right) X_{v}+O(1) m \beta_{m} X_{m} \\
= & O(1) \sum_{v=1}^{m-1} v\left|\Delta \beta_{v}\right| X_{v}+O(1) \sum_{v=1}^{m-1} \beta_{v} X_{v}+O(1) m \beta_{m} X_{m}=O(1) \text { as } m \rightarrow \infty .
\end{aligned}
$$

Since $\Delta\left(\frac{P_{v}}{v p_{v}}\right)=O\left(\frac{1}{v}\right)$, as in $J_{n, 1}$, we obtain

$$
\begin{aligned}
\sum_{n=2}^{m+1} \varphi_{n}^{\delta k+k-1}\left|J_{n, 3}\right|^{k} & =O(1) \sum_{n=2}^{m+1} \varphi_{n}^{\delta k+k-1}\left(\frac{p_{n}}{P_{n} P_{n-1}}\right)^{k}\left(\sum_{v=1}^{n-1} P_{v}\left|s_{v}\right|\left|\lambda_{v}\right| \frac{1}{v}\right)^{k} \\
& =O(1) \sum_{n=2}^{m+1} \varphi_{n}^{\delta k-1} \frac{1}{P_{n-1}^{k}}\left(\sum_{v=1}^{n-1} \frac{P_{v}}{p_{v}} p_{v}\left|s_{v}\right|\left|\lambda_{v}\right| \frac{1}{v}\right)^{k} \\
& =O(1) \sum_{n=2}^{m+1} \varphi_{n}^{\delta k-1} \frac{1}{P_{n-1}}\left(\sum_{v=1}^{n-1}\left(\frac{P_{v}}{v p_{v}}\right)^{k} p_{v}\left|s_{v}\right|^{k}\left|\lambda_{v}\right|^{k}\right)\left(\frac{1}{P_{n-1}} \sum_{v=1}^{n-1} p_{v}\right)^{k-1} \\
& =O(1) \sum_{n=2}^{m+1} \varphi_{n}^{\delta k-1} \frac{1}{P_{n-1}} \sum_{v=1}^{n-1}\left(\frac{P_{v}}{v p_{v}}\right)^{k-1} \frac{P_{v}}{v p_{v}} p_{v}\left|s_{v}\right|^{k}\left|\lambda_{v}\right|^{k} \\
& =O(1) \sum_{v=1}^{m} \frac{P_{v}}{v p_{v}} p_{v}\left|s_{v}\right|^{k}\left|\lambda_{v}\right|^{k} \sum_{n=v+1}^{m+1} \varphi_{n}^{\delta k-1} \frac{1}{P_{n-1}} \\
& =O(1) \sum_{v=1}^{m} \frac{P_{v}}{v p_{v}} p_{v}\left|s_{v}\right|^{k}\left|\lambda_{v}\right|^{k-1}\left|\lambda_{v}\right| \varphi_{v}^{\delta k} \frac{1}{P_{v}} \\
& =O(1) \sum_{v=1}^{m} \varphi_{v}^{\delta k} \frac{\left|s_{v}\right|^{k}}{v}\left|\lambda_{v}\right|=O(1) \text { as } m \rightarrow \infty,
\end{aligned}
$$

by means of (1), (4), (6), (8)-(10) and (12).

Finally, as in $J_{n, 3}$, we have

$$
\begin{aligned}
\sum_{n=2}^{m+1} \varphi_{n}^{\delta k+k-1}\left|J_{n, 4}\right|^{k} & =O(1) \sum_{n=2}^{m+1} \varphi_{n}^{\delta k+k-1}\left(\frac{p_{n}}{P_{n} P_{n-1}}\right)^{k}\left(\sum_{v=1}^{n-1} P_{v}\left|s_{v}\right|\left|\lambda_{v}\right| \frac{1}{v}\right)^{k} \\
& =O(1) \text { as } m \rightarrow \infty
\end{aligned}
$$

in view of (1), (4), (6), (8)-(10) and (12).

Thus, the proof of Theorem 2 is completed.

\section{CONCLUSION}

If we take $\left(X_{n}\right)$ as a positive non-decreasing sequence, $\varphi_{n}=\frac{P_{n}}{p_{n}}$ and $\delta=0$ in Theorem 2, then we get Theorem 1. In this case, condition (10) reduces to condition (5). Also, the conditions (8) and (9) are automatically satisfied. 


\section{REFERENCES}

[1] Bari N. K., Stečkin S. B. Best approximations and differential properties of two conjugate functions. Trudy Moskov. Mat. Obšč. 1956, 5, 483-522. (in Russian)

[2] Bor H. On two summability methods. Math. Proc. Camb. Philos. Soc. 1985, 97, 147-149.

[3] Bor H. A note on $\left|\bar{N}, p_{n}\right|_{k}$ summability factors of infinite series. Indian J. Pure Appl. Math. 1987, 18, 330-336.

[4] Bor H. On local property of $\left|\bar{N}, p_{n} ; \delta\right|_{k}$ summability of factored Fourier series. J. Math. Anal. Appl. 1993, 179 (2), 646-649.

[5] Bor H., Seyhan H. On almost increasing sequences and its applications. Indian J. Pure Appl. Math. 1999, 30, 1041-1046.

[6] Bor H., Özarslan H. S. On absolute Riesz summability factors. J. Math. Anal. Appl. 2000, 246, 657-663.

[7] Bor H., Özarslan H. S. A note on absolute summability factors. Adv. Stud. Contemp. Math. (Kyungshang) 2003, $6(1), 1-11$.

[8] Hardy G. H. Divergent Series. Oxford University Press, Oxford, 1949.

[9] Karakaş A. A note on absolute summability method involving almost increasing and $\delta$-quasi-monotone sequences. Int. J. Math. Comput. Sci. 2018, 13 (1), 73-81.

[10] Kartal B. On generalized absolute Riesz summability method. Commun. Math. Appl. 2017, 8 (3), 359-364.

[11] Mazhar S. M. A note on absolute summability factors. Bull. Inst. Math. Acad. Sinica 1997, 25, $233-242$.

[12] Mishra K. N., Srivastava R. S. L. On $\left|\bar{N}, p_{n}\right|$ summability factors of infinite series. Indian J. Pure Appl. Math. 1984, 15, 651-656.

[13] Özarslan H. S. On almost increasing sequences and its applications. Int. J. Math. Math. Sci. 2001, 25, $293-298$.

[14] Özarslan H. S. A note on $\left|\bar{N}, p_{n} ; \delta\right|_{k}$ summability factors. Indian J. Pure Appl. Math. 2002, 33, 361-366.

[15] Özarslan H. S. On $\left|\bar{N}, p_{n} ; \delta\right|_{k}$ summability factors. Kyungpook Math. J. 2003, 43, 107-112.

[16] Seyhan H. On the local property of $\varphi-\left|\bar{N}, p_{n} ; \delta\right|_{k}$ summability of factored Fourier series. Bull. Inst. Math. Acad. Sinica 1997, 25, 311-316.

[17] Seyhan H. A note on absolute summability factors. Far East J. Math. Sci. 1998, 6 (1), 157-162.

[18] Seyhan H. On the absolute summability factors of type (A,B). Tamkang J. Math. 1999, 30 (1), $59-62$.

[19] Seyhan H., Sönmez A. On $\varphi-\left|\bar{N}, p_{n} ; \delta\right|_{k}$ summability factors. Portugaliae Math. 1997, 54, $393-398$.

Received 21.02.2019

Каракас А. Нова факторизачійна теорема для узагальненого абсолютного підсумовування за Рісом // Карпатські матем. публ. - 2019. - Т.11, №2. - С. 345-349.

Метою цієї статті $є$ розгляд методу абсолютного підсумовування і узагальнення теореми про $\left|\bar{N}, p_{n}\right|_{k}$ сумовність нескінченного рялу до $\varphi-\left|\bar{N}, p_{n} ; \delta\right|_{k}$ сумовності, використовуючи майже зростаючі послідовності. Більше того, показано, що добре відомі результати для $\left|\bar{N}, p_{n}\right|_{k}$ сумовності випливають з цих узагальнень за деяких обмежень.

Ключові слова і фрази: сумовні дільники, майже зростаюча послідовність, ряди, нерівність Гьольдера, нарівність Мінковського. 